\title{
Developing a complex intervention whilst considering implementation: the TANDEM (Tailored intervention for ANxiety and DEpression Management) intervention for patients with chronic obstructive pulmonary disease (COPD)
}

Liz Steed ( $\nabla$ e.a.steed@qmul.ac.uk)

Queen Mary University of London https://orcid.org/0000-0003-1926-3196

Karen Heslop-Marshall

Newcastle Upon Tyne Hospitals NHS Foundation Trust

\section{Ratna Sohanpal}

Queen Mary University of London

\section{Sarah Saqi-Waseem}

University College London Hospitals NHS Foundation Trust

Moira Kelly

Queen Mary University of London

Hilary Pinnock

The University of Edinburgh

Stephanie Taylor

Queen Mary University of London

\section{Research}

Keywords: Depression, Anxiety, Cognitive Behavioural Therapy (CBT), Self-Management, Chronic Obstructive Pulmonary Disease (COPD), Implementation, Intervention development

Posted Date: January 10th, 2021

DOI: https://doi.org/10.21203/rs.3.rs-35082/v2

License: (c) (1) This work is licensed under a Creative Commons Attribution 4.0 International License. Read Full License

Version of Record: A version of this preprint was published at Trials on April 6th, 2021. See the published version at https://doi.org/10.1186/s13063-021-05203-x. 


\section{Abstract}

Background Guidelines now call for a thorough and comprehensive description of the development of healthcare interventions to aid evaluation and understanding of the processes of change. This was the primary aim of this study but we also recognized that effective interventions are commonly not implemented in clinical practice. It is suggested that insufficient attention is given to the implementation process at the development phase of interventions. This study outlines the 5 step iterative process we adopted for considering both implementation and effectiveness issues from the outset of intervention development. We use the development of a complex intervention Tailored intervention for ANxiety and DEpression Management (TANDEM) in patients with chronic obstructive pulmonary disease to illustrate this process.

Methods Intervention development built upon the Medical Research Council framework for developing complex interventions and the Person Based Approach for development of behavioural interventions. Building an expert team, specifying theory, qualitative data collection and pre-piloting were all critical steps in our intervention development and are described here.

Results Contact with experts in the field, and explicitly building on previous work, ensured efficiency of design. Qualitative work suggested guiding principles for the intervention such as introducing mood in relation to breathlessness, and providing flexible tailoring to patients' needs, whilst implementation principles focused on training selected respiratory professionals and requiring supervision to ensure standards of care. Subsequent steps of intervention development, pre-piloting and intervention refinement led to an intervention that was deemed acceptable and if successful will be ready for implementation.

Conclusions The TANDEM study was developed efficiently by building on previous work and considering implementation issues from the outset, with the aim that if shown to be effective it will have more rapid translation in to the health care system with accelerated patient benefits.

Trial Registration ISRCTN, ID: ISRCTN59537391. Registered on 20 March 2017. Protocol version 6.0, 22 April 2018.

\section{Contributions To The Literature}

- We describe a five-step iterative process to develop the TANDEM intervention considering both effectiveness and implementation strategies throughout.

- We engaged an expert team and built upon previous work to ensure an efficient approach to intervention design.

- The approach builds on and integrates the Medical Research Council complex intervention framework and the Person Based Approach to intervention development, and includes both health care professional and patient input from the outset. 
- The study illustrates how testing of the intervention as a whole in the development phase can be used to promote effectiveness and develop implementation strategies prior to formal piloting of the trial processes.

\section{Background}

In recent decades the science of developing complex interventions has made significant strides forwards with publication of guidelines and frameworks (1-3). Recognised methods for categorising and describing interventions have become available e.g. the Template for Intervention Description and Replication checklist TIDieR (4), the Workgroup for Intervention Development Recommendations (WIDER) (5) and most recently, for describing intervention development GUldance for the reporting of Intervention Development GUIDED (6). These methods are recommended with a view to enabling replication by healthcare services. Despite this, there is commonly a gap between an intervention being shown to be clinically and cost effective and its implementation into practice $(7,8)$. Reasons for this will be multifactorial but include organisational factors not being considered sufficiently at the outset, or a design which does not lend itself to delivery as intended (fidelity) in clinical practice. This is increasingly leading to multiple research phases where an effectiveness trial is followed by development and evaluation of implementation strategies $(9,10)$.

This trajectory of stepwise development is costly, inefficient, and may delay attainment of beneficial health outcomes. Recent approaches that may improve the process of complex intervention development include building and improving upon existing interventions, using a collaborative approach to intervention development, and considering implementation issues (including issues enhancing fidelity) at the outset (11).

The current article uses the development of a complex intervention, TANDEM for patients with moderate to very severe chronic obstructive pulmonary disease (COPD) and mild to moderate anxiety and/or depression, to illustrate how we built on existing innovations, involved expert stakeholders in a collaborative team approach and considered implementation throughout the process. Our dual aims were to optimise effectiveness and also implementation of the intervention, if it is shown to be effective.

\section{Managing Chronic Obstructive Pulmonary Disease (COPD)}

COPD has a global prevalence of $11.7 \%$ in adults aged over 30 years (12) and is associated with substantial morbidity and mortality (13) with indications that it will be among the top three causes of death by 2030 (14). Progressive reduction in lung function and increased breathlessness affect the physical, social and emotional worlds of patients (15). People with COPD typically have multi-morbidity, 
including psychological conditions (16-18), such as anxiety and depression, which have a major influence on quality of life $(19,20)$. Anxiety is reported across all ranges of COPD severity, with cited prevalence ranging from 10 to $50 \%$ depending on definitions and populations studied $(21,22)$. The prevalence of depression (on average around $30 \%$ of all COPD patients $(21,23)$ ) increases with the severity of COPD (24). Importantly people with COPD and anxiety/depression experience more exacerbations, more frequent and longer hospital admissions, and reduced survival $(22,25-28)$.

Pulmonary rehabilitation (PR) is an evidence-based, guideline-recommended intervention for the management of COPD (29), which improves functional capacity, psychological well-being, and quality of life (29-31). However, uptake and reach of PR is less than optimal (32) as practitioners under-refer, and patients decide not to, or are unable to, attend, or complete, their PR course (33) which may be in part due to co-existing anxiety and/or depression (34). Psychological interventions using a cognitive behaviour therapy (CBT) approach either alone, or as a component of PR, have shown promise $(30,35,36)$. However, the limited evidence to date, has not considered implementation issues such as reach, or the practicalities of workforce delivery. TANDEM was developed to address this research gap and specifically to design an implementable, cognitive behavioural approach (CBA) intervention, delivered in tandem with PR, aiming to both improve symptoms of anxiety and depression and increase uptake and completion of PR, which itself further improves psychological well-being. The protocol for the TANDEM trial is published (37). By CBA we recognise that our intervention does not deliver full CBT but rather draws on the underlying theory to deliver an approach based on CBT. From the outset the process of developing the intervention was understood to be complex, and we kept an open mindset to different guidance and methodologies that we would need. This has subsequently been distilled into a five-step iterative process which is described below and illustrated in Figure one. Throughout these steps we also worked closely with our PPI co-applicant, who had been instrumental in designing the original grant proposal and remained as an expert by experience throughout the development of the intervention.

\section{Methods}

Step One - Building an expert study team

Development of TANDEM arose out of a commissioned funding bid for a tailored psychological intervention "combined with physical retraining" for individuals with anxiety and or depression and moderate to severe COPD. A review cited within the commissioning brief (36) suggested there was synergy between psychological approaches and physical activity in improving COPD. Proposals were requested to meet this need. In order to design and deliver such an intervention the first step was building a multidisciplinary team with relevant expertise. The expertise that was deemed necessary included individuals with experience in: respiratory medicine, pulmonary rehabilitation, cognitive behavior therapy in COPD, self-management support in long term conditions, educationalists, health and clinical psychology, trials and research methodology, qualitative methodology and the lived experience of COPD 
(PPI). Individuals who the principal investigators (SJCT and HP) knew in each of these field were invited to participate in the expert team.

The expert team met at each stage of the intervention development process to suggest content, consider theory, review feedback and revise the intervention.

Step Two - Developing an outline of the intervention and consideration of theory

Based on the experience of the expert team suggestions for an initial outline intervention (see supplementary file 1) were made. The theory underpinning these previous interventions was then identified.

Theory use was recommended at multiple levels with multiple objectives (e.g. to guide the intervention development process, to inform the patient facing element of the intervention, to inform training in delivery of the facilitator facing intervention). Selection of specific theory was guided by those with which the expert team had experience, and which were supported by the literature. Implementation theories were not considered at this point as the plan was to initially understand implementation issues (in line with a hybrid type 1 approach (38) and then be directed to an appropriate theory as part of the process evaluation (39).

Given the theoretical complexity of the intervention we developed a programme theory and a logic model showing how we envisaged the different approaches would work together and complement each other in the overall TANDEM intervention.

Step Three - Qualitative research to understand participant and implementation needs and develop guiding principles

Building on the expert teams past experience, and materials and theory from steps one and two, understanding 'real life' challenges for patients, health care professionals and health systems, was considered essential to inform an implementable intervention. We therefore conducted exploratory qualitative work with both health care professionals and patients with topic guides that addressed three issues:- 
1. Difficulties in living with COPD

2. Opinions on a preliminary outline intervention (developed in step two)

3. Critical elements for successful implementation

We identified respiratory health care professionals (rHCPs) who had an interest in delivery of psychological interventions to patients with COPD, through social media and professional networks, and invited them to participate in either an individual interview (face to face or telephone) or a focus group, dependent on participant preference.

We arranged two focus groups for patients and carers. One included COPD patients or carers who had previously experienced CBT (including participants from a specific CBT trial (40); and one with patients and carers attending a Breathe Easy group (a UK support group run by the British Lung Foundation (BLF)) who may, or may not, have experienced CBT. Our PPI expert supported us in approaching and designing these focus groups and interpretation of the results.

Informed consent to participate and audio-record data was obtained for all participants. Recordings were then transcribed. As the purpose of this qualitative work was to inform intervention development we conducted a rapid thematic analysis using a framework approach (41), with the key aim of identifying guiding principles that should be included in the intervention to increase acceptability and ease implementation into routine service if shown to be effective.

Step Four - Detailed design of intervention materials and mode of delivery

KHM and LS reviewed all feedback on the outline intervention and issues around delivery from step three and the guiding principles for the intervention and its implementation (see supplementary material 2). These were used to develop more detailed content of the intervention. In addition, at this stage, we were guided by recommendations for enhancing fidelity in behavioural interventions (42).

A reflective intervention development log was kept to ensure the process, considerations and decisions taken were recorded and transparent (see supplementary material 2).

Step Five - Whole intervention pre-pilot testing 
Given the complexity and multi-level action of the TANDEM intervention it was felt that even after individual materials had been developed and refined the intervention needed to be delivered as a whole, to understand how elements 'hung' together. This was seen as an explicit part of the design phase of the intervention, and an important step prior to testing any research elements (such as randomisation, or outcome data collection) that was scheduled for a pilot trial (43). We identified two key questions which needed to be answered before formal piloting:-

1. Do participants who receive the intervention when delivered by someone already skilled in CBT find it acceptable (i.e. is the potential TANDEM patient facing intervention appropriate and acceptable) and receive the intervention as intended (delivered with fidelity)?

2. Do participants who receive the intervention when delivered by a novice in a cognitive behavioural approach (CBA,) but trained as part of the TANDEM programme, find the intervention acceptable and receive it as intended (i.e. is the proposed TANDEM facilitator training sufficient for delivery of the TANDEM patient intervention and consequently implementable)?

A purposive sample of three rHCPs were recruited and trained to deliver the TANDEM intervention, these included one rHCP trained to CBT diploma level, one trained to basic CBT level (i.e. following a three day training external to TANDEM) and one rHCP who had not previously received any CBT training. Once trained in the TANDEM intervention these individuals were referred to as "TANDEM facilitators".

As part of the facilitator-facing intervention, training for the TANDEM facilitators was conducted over three days, the first two concurrent and the third approximately 6 weeks later to enable practice of skills. To reflect the group nature of future training, clinical members of the research team also joined the three days training as participant observers. Training was delivered by a CBT qualified consultant respiratory nurse (KHM) a health psychologist (LS) and a consultant clinical psychologist (SSW). At the end of each training day all participants were requested to provide verbal feedback on the content and process of training.

Patients with moderate to severe COPD were eligible for participation in the whole intervention pre-pilot phase. Patients received the full 6-8 TANDEM sessions and were invited to interview post-intervention. With participant consent all intervention sessions were audio-recorded. Following delivery of the patient intervention all three of the rHCP TANDEM facilitators were invited to interview. Semi structured interviews were conducted and covered issues such as acceptability and benefit of training, any omissions or improvements, feasibility of delivering the intervention to patients and usefulness of intervention materials. 


\section{Results}

Step One - Building an Expert Team

Dr Karen Heslop Marshall, a clinical respiratory academic who had experience of delivering CBT in COPD agreed to be part of the expert team, as did Professor Sally Singh a pulmonary rehabilitation expert. Education for Health, a health education charity, were approached and agreed as did psychologists Dr Liz Steed and Dr Sarah Saqi-Waseem. Chris Warburton was an expert patient who joined the team and offered PPI consultation throughout. Methodologists included Moira Kelly and Ratna Sohanpal, who both had experience of working in the field of COPD.

This expert team proposed combining CBA and a supportive self-management intervention with exercise, provided by explicitly linking in to routine $\mathrm{PR}$, in order to build upon an evidence-based service already embedded within the NHS. The team proposed building on two pre-developed interventions The Lung Manual (44) and the SPACE Manual (45) including use of their materials e.g. SPACE materials as well as the full range of information leaflets for people with COPD and their carers from the British Lung Foundation to meet educational and self-management needs. This meant materials which had already had extensive PPI input were available. We also drew on CORE competencies for delivering CBT (46)

Step Two - Use of theory to develop a logic model and preliminary outline of the intervention

Both The Lung Manual and the SPACE manual are evidence-based and draw on theory. The Lung Manual applies Beck's theory of CBT (47) for managing anxiety and breathlessness in COPD whilst the SPACE manual applies a self-management approach based on Bandura's social learning/cognitive theory $(48,49)$. These theories were therefore considered important underpinnings for TANDEM. LS suggested that Leventhal's Self-Regulation theory (50) may also be relevant to self-managing COPD. This was supported by the literature and therefore incorporated in the programme theory for patient-facing aspects of the intervention.

Consultation with Education for Health suggested that the pedagogical theory that would be most relevant for training facilitators was the VARK (Visual, Auditory, Read, Kinesthetic (i.e. experience or practice, simulated or real)) model of learning (51). This has been used frequently in training interventions and ensures that the training suits individuals with different learning styles and we adopted it in the current study. 
For intervention development the person-based approach (52) with its focus on using qualitative work to inform guiding principles was considered particularly relevant and therefore guided the intervention development process.

Figure two shows the logic model for TANDEM. The basic premise is that how an individual thinks about their COPD (cognitions - including illness and treatment beliefs) influences how they behave (including self-management actions taken) and how they feel (both physical symptoms and emotions). These factors have interactional effects such that depression and /or anxiety can be both reduced or exacerbated depending on the individual's cognitions and behaviours. Consequently, by targeting change at a cognitive, behavioural or symptom level this will influence emotional outcomes. This improvement in emotional outcomes and self-management outcomes is then hypothesised to make attendance at pulmonary rehabilitation more likely, which itself is known to have positive outcomes including on both physical and psychological outcomes(30,31).

Based on the logic model and content from the Lung and SPACE manuals a preliminary outline intervention for discussion in step three was developed (see supplementary file one). This included information on COPD including illness and treatment beliefs, and skills such as breathing techniques; cognitive behavioural techniques such as monitoring, diaries, distraction; basic self-management techniques such as goal setting and problem solving; delivery options were open as were the best ways to train facilitators and who those facilitators should be and other factors which would support implementation

Step Three - Qualitative findings, themes and development of guiding principles

One focus group comprising six rHCPs (one respiratory consultant, one occupational therapist, three physiotherapists and one exercise practitioner) and seven individual interviews (four psychologists, two physiotherapists and one general practitioner) were conducted. All participants had experience in working with patients with COPD, either in the community or secondary care. Roles varied, including some with management responsibilities who were able to discuss implementation.

One focus group was held with patients of whom four had COPD, two had other respiratory conditions and two were carers. Very tight timelines precluded formal analysis of transcripts from the patient focus group data, so data were limited to quotes selected from the audio recording. Major local governance 
delays prevented conduct of the second focus group with patients and their carers who had experience of $\mathrm{CBT}$ in time to inform intervention development.

Both patients and professionals presented an overall positive attitude to the idea of the TANDEM intervention:-

And I do think in the long run something like this could be more cost effective and stuff, things like that...I think it would be really useful (rHCP FG002 Occupational therapist)

Themes were developed which related to i) life with COPD, ii) intervention considerations and iii) issues for implementation.

Life with COPD

All participants, including patients and rHCPs, recognised depression and anxiety as common in COPD although patients did not always use this terminology. Other issues such as frustration and embarrassment, along with role adjustment and loss, including of social contacts, were common and seen as contributors to mood problems.

"The approach is good...because of all the other things happening in people's lives which can stop them attending PR and make them anxious and depressed." (focus group patient)

Intervention considerations

rHCPs emphasised the challenge of breathlessness to patients with COPD and suggested that discussion and teaching of breathing control early in the intervention would be a helpful way to raise issues around mood as well as providing practical help which may increase engagement. There was also recognition that this group may be quite socially isolated and health literacy may vary so the intervention must be accessible to all.

Implementation considerations 
The majority of issues for implementation related to the workforce and who would realistically be able to deliver a CBA service. Both rHCPs and psychologists recommended rHCPs to be best placed, however all recognised that there would need to be some selection process and supervision. One clinician was concerned about the cost of the intervention, although others considered that in the long run CBA could be cost-effective.

These themes were subsequently interpreted to provide guiding principles as recommended by the patient-based approach to intervention development (52). Table 1 shows these principles and example data extracts.

Step Four: Detailed design of intervention materials and mode of delivery

Having agreed the guiding principles for the intervention the expert team met to discuss the detailed design of the TANDEM intervention. It was understood that the intervention would be working at two levels i) patient-facing (i.e. CBA delivery) and ii) facilitator-facing (i.e. training programme).

For the patient-facing CBA a range of core patient self-completion materials were designed that could be provided as part of the intervention. These covered the topics of 'Controlling your breathing', 'Mood and COPD', 'Anxiety and COPD', 'Depression and COPD', 'Problem Solving', 'Saving Energy'. These were developed for TANDEM but where possible drew on, or used, the SPACE manual (46) handouts and were of similar format to published CBT leaflets accessible on the internet (53) as these have been developed with extensive PPI. The aim of these leaflets was as homework, (called "home practice" in the TANDEM intervention based on PPI advice that connotations of school may be off putting for people who had poor experiences of school), which is a central part of CBT and to reinforce knowledge that had been covered in the one-to-one sessions. The approach is also in line with (Integrated Access to Psychological Therapies (IAPT)) services low-intensity provision (54). LS developed all materials with iterative refinement from the expert team including PPI. At the start of session one each patient was given a TANDEM folder, in which they could store handouts relevant to them so that individuals had a tailored version of TANDEM materials whilst maintaining consistency in the content provided. In total 6-8 face to face sessions were designed, covering nine topics; with core content and additional modules tailored to individual problems and complexity. Table two provides an overview of TANDEM topics

One topic (dealing with 'other problems') was specifically added as a strategy for keeping the focus of initial sessions on COPD whilst having space later to address issues the person may have outside COPD, for example debt, substance abuse etc. This topic looked at how the CBA, learnt in the context of COPD, 
could be generalised to different problems, with sign-posting to additional sources of help. At the final session discussion was around pulmonary rehabilitation. If there was to be a delay in individuals commencing PR then the facilitator arranged up to nine weekly telephone calls.

The three-day facilitator training was provided with a supporting manual which covered the skills needed to deliver TANDEM.

Table 3 shows the content of the training programme. There was a high level of practical and experiential learning in the group and supportive links within the group were encouraged. Throughout delivery of the CBA intervention facilitators received regular telephone supervision, one-to-one with senior cognitive behavioural therapists at approximately fortnightly intervals. This on-going supervision was considered to be an integral part of the intervention.

Phase Five - Whole intervention pre-pilot study

All three TANDEM facilitators completed the three training days and two went on to deliver the intervention to three patients (one delivered it to two patients and one to a single patient). The third facilitator (a respiratory practice nurse without prior CBT training) did not manage to see any patients due to unanticipated research governance delays and a consequent change in work commitments.

\section{TANDEM Facilitators}

After the initial training session, facilitators suggested some changes (see supplementary file two), specifically an overview of the intervention at the beginning of the training in order to orientate individuals. All the facilitators felt that the role-play activity with a simulated patient (actor), which was conducted as part of the original group training on day one, was too threatening and at too early a stage of skill development. Instead they requested more demonstrations and more practice in developing a formulation.

Interviews at the end of delivering TANDEM to patients, revealed that both facilitators felt the intervention had been well received by participants and feasible to implement, although one had to deliver it over a longer period than scheduled due to patient illness.

'Yeah, I mean the two patients who I had were very, very enthusiastic about all elements of the intervention. (PPHCP01)' 
Generally, the facilitators appeared able to follow the manual and found it a helpful guide, but there was questioning of whether someone without previous CBA training would be able to manage:

'I mean section nine, it's got identifying maintenance factors, and it talks about safety behaviours, avoidance and escape, catastrophic interpretation, scanning or hypervigilance, self-fulfilling prophecies, fear of fear, reductions, affectionism, short term rewards. If you're trying to talk to a patient and remember what it says in the manual you might get yourself a little bit flustered.' (PPHCP02)

One facilitator recommended presenting basic intervention techniques as a toolbox and also the provision of a crib sheet for easy prompting within sessions.

'I feel that people who come away from the training need to have something like a virtual toolbox of techniques that they can refer to ...they expected quite a lot of you... I made myself a crib sheet type of thing' (PPHCP01)

One element that was not adhered to as planned was supervision with a senior psychologist, as the facilitators relied on supervision by an experienced member of their team who was already known to them and who was also part of the study team (KHM). However, both facilitators reported this supervision was useful.

\section{Patient perspective}

Patients who had received the TANDEM intervention reported it to be acceptable and beneficial, observing that the facilitators had very good interpersonal skills. There were no substantive suggestions for improvement.

'And then [facilitator] and I just seemed to get on very well, he's a likeable chap, very laid back. And so it went from there. And then we started doing the things that you asked in TANDEM. Planning... They're just small things, but marvelous' (PPP01, male participant) 
The findings, such as patients reporting activities like planning (see quote above), and facilitators commenting on applicability of acceptance exercises suggested that the facilitators delivered the intervention with fidelity, however this needs to be explored in more detail through use of for example audio-recordings of sessions. This is planned for the process evaluation in the pilot and main trial (39)

\section{Refinements to TANDEM after the pre-pilot}

Changes and additions were primarily made to the TANDEM facilitator training, as there were few recommendations for changes to patient materials. These are shown in Table 3 with additions highlighted in bold. All suggestions were followed: e.g. providing a greater overview of TANDEM at the beginning of session one, keeping to a core set of CBA techniques and outlining a "toolbox" of techniques which could be used, (literally presented like a tool box in the revised manual). We made video recordings to demonstrate therapeutic skills and CBA techniques. These were made available online, with a facilitator chat facility for ongoing support.

The use of a simulated patient was omitted from the first two days of training and replaced by partnered role-play. The simulated patient role-play was, however, added to the end of day three. Each TANDEM facilitator was individually video-recorded conducting a cognitive behavioural assessment and feedback

with the actor. Each video was subsequently assessed by LS and at least one independent assessor with a psychology background to ensure that a minimum standard of competency (see study protocol(37)) had been acquired. This also enhanced fidelity of delivery. To enhance learning, and boost confidence, facilitators received one-to-one feedback on their video. A training session on the importance of supervision was added with reflection that supervision is a standard part of psychological training and practice (in contrast to more managerial supervision with which HCPs may be more familiar).

\section{Refinements to improve implementation}

To improve delivery of the intervention within the trial and future implementation within routine healthcare contexts, five features were added:

1. Facilitators were provided with crib cards for use as prompts within sessions.

2. An optional session was added for use when a break in sessions had become necessary (e.g. due to patient illness) in order to refresh topics that had been covered before the break and re-establish current priorities.

3. Some flexibility in the order of delivery of sessions was allowed reflecting the reality that some patients commenced PR before the end of the TANDEM sessions. It was stipulated that Topics 1-5(or 
6) must have been conducted but that if necessary the final topic on expectations of PR could be brought forward as there was no sense in delivering this once PR had started.

4. A structure for screening potential facilitators including a formal application with a curriculum vitae and telephone interview with one of the principal investigators was developed. The aim was to ensure only fully committed individuals who were interested in the psychological aspects of the experience of living with COPD and who could meet the study-specific requirements (e.g. flexibility to travel, willingness to complete research modules and good clinical practice training) received training. Also facilitators needed to be made aware that training involved some role play and that all intervention sessions would be recorded for fidelity assessments.

5. A booster training session was designed to be delivered to facilitators if there were delays of 3 months or more between initial training and delivery of TANDEM.

For a description of the intervention following TIDieR guidance please see additional file 1.

\section{Discussion}

This paper describes the development of the TANDEM intervention for COPD, that considers both intervention and implementation strategies from the outset, with the aim of reducing the time period for translation of the intervention (if successful) into practice. In particular, consideration was given to the workforce that would deliver the intervention and their training and support needs, as well as designing an intervention that could fit structurally into routine clinical care. This would also make it more likely the intervention could be delivered with fidelity.

An important element of development was the whole intervention pre-pilot testing. This is particularly applicable for complex multi-level or group interventions where the recommended 'think aloud' phase (52) of development may be impractical. The benefits of a pre-pilot phase are to test not only individual elements of an intervention but also how multiple elements of an intervention 'hang together'. Similar recommendations have been made by others such as in the ORBIT Model (55). Thorough testing in the development phase is important and should come before a pilot trial which can then focus more on testing elements of the research process (43). Further testing of acceptability can still be done when piloting the intervention, as is planned for the TANDEM intervention (see (39)), however the fewer changes that need to be made further along the evaluative trajectory the more efficient evaluation of the intervention is likely to be.

Whilst we present a stepped process in reality there was flow between different steps as intervention development is commonly iterative (2). For example, a preliminary outline of the intervention had been 
developed in step two before the guiding principles were articulated in step three. This was inevitable as our initial design was reactive to a funding call (with the additional benefit of enabling peer review comment on our ideas). Importantly, however, at step three we were open to any of our original ideas being challenged and the intervention changed. The expert study team comprised individuals with diverse backgrounds and were encouraged to reflect and discuss critically.

A challenge we recognised from the outset was that for interventions to be implemented within routine health care services they must be as cost effective as possible. One implication of this is that each patient should receive the optimal intervention for them; one size fits all interventions are likely to be cost inefficient either by under or over treating some individuals. Tailoring has been recommended as a way to address this (7), though it is a significant challenge to design an intervention that is both responsive to each individual's needs yet standardised enough to allow for robust evaluation and assessment of fidelity when implemented. TANDEM attempted to balance standardisation with flexibility by using a modular/topic-based approach where topics are standardised, but which topics are addressed and in what order is dependent on the individual or the circumstances (such as timing of the PR course). The success of tailoring in this way is as yet unknown but will be examined as part of the process evaluation of the trial as will fidelity.

A further challenge was that some aspects of implementation were more difficult because of delivery within the context of a research trial. In our pre-pilot we experienced two illustrations of this. The first was that obtaining local research governance approval caused a delay of 6 months between training TANDEM facilitators and being allowed to deliver the intervention (arising when a local research governance office unexpectedly changed its classification system). This was detrimental as it risked loss of confidence amongst the facilitators, skill drift and knowledge decrease during the gap between training and implementation. We would urge research governance frameworks to consider the impact of their processes in the context of intervention development and implementation research. Within a clinical service this might be less likely to occur, but clinical practice has its own challenges such as staff turnover, funding pressures, changes in management etc. The implementation strategies we provided, such as booster training and online support resources to overcome study specific issues, could equally apply in the clinical setting and will be explored within our trial process evaluation.

The second was that our TANDEM facilitators could not be embedded within the participants' clinical team (to prevent 'contamination' of controls (37)) with the results that they would not have support, both practical and emotional, from clinical colleagues. The research team attempted to overcome this by providing facilitators with a clinical support network with the chief investigators ST, HP (both general practitioners) and the consultant respiratory nurse (KHM) was available to answer any concerns. This 
was in addition to the clinical supervision provided for ensuring therapeutic competence and access to a chat facility with other facilitators on the skills website.

\section{Strengths and Limitations}

A strength of TANDEM is in its multidisciplinary expert team approach to intervention development with PPI representation within that team so embedded throughout all our work. We specifically built upon previous work by collaborating with national experts enhancing the expertise available to TANDEM and reducing potential duplication. Surprisingly this has not been explicitly recommended in recent guidance (11) but in our view is an important strategy for ensuring the most efficient use of limited resources.

A further strength is the systematic and transparent approach to intervention development that we have outlined.

In step three where we conducted qualitative work to highlight important issues for the intervention and its implementation, only a limited number of health care professionals and patients could participate. It may be that important voices such as that of practice nurses, health service managers or commissioners, who could have provided different perspectives for implementation, were not heard. This step, however was scheduled within a relatively rapid time-frame to be useful to inform intervention development, and the themes will be further explored in the pilot/trial process evaluation.

In the pre-pilot phase only three facilitators and three participants experienced the intervention. Greater numbers would have provided greater feedback however again this was not possible due to resource limitations and logistical requirements of the time-scale required for intervention development. Similarly, due to time constraints we were also not able to fully evaluate fidelity, however this is planned for the pilot and main trial evaluations (39) and is an important part of intervention development.

Although we aimed to address integration of implementation issues throughout our intervention development process we did not explicitly do this in our research design and hence have not presented a logic model of implementation which will be presented following process evaluation. Methodological advances in implementation research have made recommendations for trial designs known as hybrid designs whereby effectiveness and implementation potential of strategies are investigated concurrently (38). Recently there have also been calls for this within behavioural science (56). Three levels of hybrid design are proposed which vary in the relative balance between the focus on effectiveness versus 
implementation (41). These designs require a set of assumptions to be met around features of the intervention, such as the level of face validity, strength of the existing evidence base, risks associated with the intervention and implementation momentum. TANDEM meets these assumptions and hence can be considered as similar to a type one hybrid design where the primary focus is on effectiveness but issues of implementation are explored for example:

1. It has high face validity given $\mathrm{CBT}$ and pulmonary rehabilitation are well recognised and guidelinerecommended interventions.

2. PR has an established evidence base for COPD (29-31) and CBT is showing promise for COPD patients $(35,43)$.

3. Few risks have been described in evaluations of CBT or PR and there is little reason to expect increased risk through the integration of these approaches in TANDEM;

4. Importantly there is currently momentum within the clinical system towards both implementation of PR (57), and treatment of psychological issues within chronic illness, in the UK (58). Furthermore recent policy has advocated the expansion of current primary care mental health services to work with patients with COPD (IAPT services) (54).

Future studies developing interventions for implementation may wish explicitly to consider a greater diversity of research design, including hybrid designs to facilitate implementation.

\section{Conclusions}

We recommend that intervention developers play greater consideration to implementation issues both at the early stages and throughout the intervention development phase. We describe five steps including building an expert team and building on previous innovations, using theory, exploring the needs of the target groups, developing prototypes and testing the whole intervention in a pre-pilot. By conducting this work within a framework of critical reflectivity we aimed to maximize efficiency of intervention design and minimise the trajectory from intervention development to implementation if shown to be effective.

\section{List Of Abbreviations}

BLF: British Lung Foundation

CBA: Cognitive Behavioural Approach

CBT: Cognitive Behaviour Therapy

COPD: Chronic Obstructive Pulmonary Disease

GUIDED: GUIDance for the rEporting of intervention Development 
IAPT: Integrated Access to Psychological Therapies

MRC: Medical Research Council

PPI: Patient and Public Involvement

PR: Pulmonary Rehabilitation

rHCP: respiratory Health Care Professional

TANDEM: Tailored intervention for ANxiety and DEpression Management

TIDieR: Template for Intervention Description and Replication

VARK: Visual Auditory Reading Kinesthetic

WHO: World Health organization

WIDER: Workgroup for Intervention Development and Evaluation Research

\section{Declarations}

Ethics approval and consent to participate

The study (pilot and main trial) was approved by the London-Queen Square Research Ethics Committee, reference 17/LO/0095. Informed consent was obtained from all study participants.

Consent for publication

Consent for publication was received from all participants before their commencement in the study.

Availability of data and materials

Data generated and analysed during this study is included in this published article. Study manuals and training materials are copyrighted but will be available upon reasonable request to the authors.

Competing interests

SJCT is the chief investigator or co-investigator on multiple previous and current research grants from the UK National Institute for Health Research and CRC UK. 
KM received funding from NIHR for a PhD study on CBT in COPD, received honoraria from pharmaceutical companies for presenting on CBT in COPD, I have received travel expenses for speaking at conferences and professional organisations on CBT and I am a director of Pivotal Health Company which provides CBT training for healthcare professionals in the physical health setting.

\section{Funding}

This report is independent research supported by the National Institute for Health Research Health Technology Assessment programme (project number 13/146/02).

SJCT is supported by the National Institute for Health Research ARC North Thames. The views expressed in this publication are those of the authors and not necessarily those of the National Institute for Health Research, the NHS or the Department of Health and Social Care.

Authors' contributions

SJCT and HP conceived the study. LS, KHM, SSW were responsible for the primary development of the intervention. All authors participated in the intervention development steering group. RS contributed to the collection of qualitative data and MK advised on analysis of qualitative data. All authors made substantial contributions to the design of the study. All authors have helped to draft this manuscript and/or revised it and all have read and approved the final manuscript.

Acknowledgements

The authors are grateful for the support of Mr. Chris Warburton and all our other Public Involvement advisors; and our Trial Steering Committee and Data Monitoring and Ethics Committee members.

\section{References}

1. Craig P, Macintyre S, et al. Developing and evaluating complex interventions: the new Medical Research Council guidance. BMJ. 2008;337.

2. O'Cathain A, Croot L, Sworn K, Duncan E, Rousseau N, Turner K, et al. Taxonomy of approaches to developing interventions to improve health: a systematic methods overview. Pilot Feasibility Studies. 2019;5:41.

3. Michie S, West R. The behaviour change wheel: a new method for characterising and designing behaviour change interventions. Implement Sci 2011;23(6). 
4. Hoffmann TC, Boutron I, et al. Better reporting of interventions: template for intervention description and replication (TIDieR) checklist and guide. BMJ. 2014;348.

5. Albrecht L, Arseneau D, Scott SD. Development of a checklist to assess the quality of reporting of knowledge translation interventions using the Workgroup for Intervention Development and Evaluation Research (WIDER) recommendations. Implement Sci 2013;8.

6. Duncan E, Rousseau N, Croot L, Sworn K, Turner KM, Yardley L, Hoddinott P. Guidance for reporting intervention development studies in health research (GUIDED): an evidence-based consensus study. BMJ open. 2020. Epub Apr 8;10(4):e033516.

7. Taylor SJ.,Pinnock H., , Epiphaniou E, Pearce G, Parke H L, Schwappach A, Purushotham N, Jacob S, Griffiths $C$ J, Greenhalgh T \& Sheikh A. A rapid synthesis of the evidence on interventions supporting self-management for people with long-term conditions (PRISMS Practical systematic Review of SelfManagement Support for long-term conditions). Health Services and Delivery Research. 2014;2(53).

8. Pinnock H, Parke, H., Panagioti, M. et al. 15, 64 (2017). Systematic meta-review of supported selfmanagement for asthma: a healthcare perspective. BMC Med. 2017;15(64).

9. Pinnock $\mathrm{H}$, Sheikh A. Standards for reporting implementation studies (StaRI): enhancing reporting to improve care. NPJ primary care respiratory medicine. 2017 Jun 26;27(1):42. PubMed PMID: 28652602.

10. Peters DH, Alonge O, Agyepong IA, Tran N. Implementation research: what it is and how to do it. BMJ. 2013;347:6753.

11. O'Cathain A., Croot L., Duncan E. et al. Guidance on how to develop complex interventions to improve health and health care. 2019. BMJ 9:8

12. Adeloye D CS, Lee C, Basquill C, Papana A, Theodoratou E, Nair H, Gasevic D, Sridhar D, Campbell H, et al. Global and regional estimates of COPD prevalence: systematic review and meta-analysis. J Glob Health. 2015;5(2): 020415

13. Soriano JB, Abate KH, Abera SF, Agrawal A, Ahmed MB, Aichour AN et al. on behalf of GBD 2015 Chronic Respiratory Disease Collaborators. Global, regional, and national deaths, prevalence, disability-adjusted life years, and years lived with disability for chronic obstructive pulmonary disease and asthma, 1990-2015: a systematic analysis for the Global Burden of Disease Study 2015. Lancet Respir Med. 2017;5(9):691-706. Epub Epub 2017 Aug 16.

14. World Health Organization. Chronic obstructive pulmonary disease Burden of Disease: . 2019.

15. Tsiligianni I, Kocks J, Tzanakis N, Siafakas N, van der Molen T. Factors that influence diseasespecific quality of life or health status in patients with COPD: a review and meta-analysis of Pearson correlations. Primary care respiratory journal : journal of the General Practice Airways Group. 2011 Sep;20(3):257-68.

16. Department of Health. An outcomes strategy for COPD and asthma: NHS Companion Document. In: Department of Health2012 Accessed 8 Oct 2019. Available from: https://http://www.gov.uk/government/publications/an-outcomes- strategy-for-copd-and-asthmanhs-companion-document 
17. Wong CJ, Marciniuk DD, Rennie D. Fatigue in patients with COPD participating in a pulmonary rehabilitation program. Int J Chron Obstruct Pulmon Dis. 2010;5:319-26.

18. Cassidy S TS, Gardani M, Kirkwood K. Attendance at pulmonary rehabilitation classes: an exploration of demographic, physiological and psychological factors that predict completion of treatment. Chron Respir Dis. 2014;11(2):95-102.

19. de Voogd JN WJ, Postema K, van Sonderen E, Ranchor AV, Coyne JC, Sanderman R. . More evidence that depressive symptoms predict mortality in COPD patients: is Type $D$ personality an alternative explanation? Ann Behav Med. 2009;38(2).

20. Papaioannou AI BK, Tsikrika S, Karakontaki F, Kastanakis E, Banya W, Haniotou A, Papiris S, Loukides S, Polychronopoulos $\mathrm{V}$, et al. . The impact of depressive symptoms on recovery and outcome of hospitalised COPD exacerbations. Eur Respir J. 2013;41(4):815-23.

21. Baxter N MV, Holzhauer-Barrie J, Robinson S, Stone P, Quint J, Roberts CM. Planning for every breath. National Chronic Obstructive Pulmonary Disease (COPD) Audit Programme: Primary care audit (Wales) 2015-17. National Report. 2017.

22. Yohannes AM, Alexopoulos GS. Depression and anxiety in patients with COPD. European respiratory review : an official journal of the European Respiratory Society. 2014 Sep;23(133):345-9.

23. Bock K BE, Hilberg O, Løkke A. Screening tools for evaluation of depression in Chronic Obstructive Pulmonary Disease (COPD). A systematic review. Eur Clin Respir J. 2017;4(1).

24. Cafarella PA ET, Usmani Z-A, Frith PA. . Treatments for anxiety and depression in patients with chronic obstructive pulmonary disease: a literature review. Respirology. 2012;17(4):627-38.

25. Ng TP NM, Tan WC, Cao Z, Ong KC, Eng P. Depressive symptoms and chronic obstructive pulmonary disease: effect on mortality, hospital readmission, symptom burden, functional status, and quality of life. Arch Intern Med. 2007;167(1):60-7.

26. Eisner MD BP, Yelin EH, Katz PP, Sanchez G, Iribarren C, Omachi TA. . Influence of anxiety on health outcomes in COPD. Thorax. 2010;65(3):229-34.

27. Laurin C MG, Bacon SL, Lavoie KL. Impact of anxiety and depression on chronic obstructive pulmonary disease exacerbation risk. Am J Respir Crit Care Med 2012;185(9):918-23.

28. Dickens C KW, Blakemore A, Khara A, McGowan L, Tomenson B, Jackson J, Walker L, Guthrie E. Does depression predict the use of urgent and unscheduled care by people with long term conditions? A systematic review with meta-analysis. J Psychosom Res. 2012;73(5):334-42.

29. McCarthy B CD, Devane D, Murphy K, Murphy E, Lacasse Y. Pulmonary rehabilitation for chronic obstructive pulmonary disease. . Cochrane Database of Systematic Reviews. 2015;2(CD003793).

30. Coventry PA, Hind D. Comprehensive pulmonary rehabilitation for anxiety and depression in adults with chronic obstructive pulmonary disease: Systematic review and meta-analysis. Journal of psychosomatic research. 2007 Nov;63(5):551-65.

31. Steiner MC, Roberts CM. Pulmonary rehabilitation: the next steps. The Lancet Respiratory medicine. 2016 Mar;4(3):172-3. 
32. McNamara RJ, Kearns R, Dennis SM, M FH, Gardner K, McDonald J. Knowledge, Skill, and Confidence in People Attending Pulmonary Rehabilitation: A Cross-Sectional Analysis of the Effects and Determinants of Patient Activation. Journal of patient experience. 2019 Jun;6(2):117-25.

33. Steiner M, Lowe D, Searle L, Skipper E, Welham S, Roberts CM. . Pulmonary Rehabilitation: time to breathe better. National Chronic Obstructive Pulmonary Disease (COPD) Audit Programme: resources and organisation of pulmonary rehabilitation services in England and Wales 2015. . 2015.

34. Sohanpal R, Steed L, Mars T, Taylor SJ. Understanding patient participation behaviour in studies of COPD support programmes such as pulmonary rehabilitation and self-management: a qualitative synthesis with application of theory. NPJ primary care respiratory medicine. 2015 Sep 17;25:15054.

35. Pollok J, Esterman AJ, Carson-Chahhoud KV. Psychological therapies for the treatment of depression in chronic obstructive pulmonary disease. . Cochrane Database of Systematic Reviews. 2019 (3):CD012347.

36. Smith SMS, Ketcheson L, Larson JL. A review of the effectiveness of psychological interventions used for anxiety and depression in chronic obstructive pulmonary disease. BMJ Open Respir Res. 2014;1(1):e000042.

37. Sohanpal R, Pinnock H, Steed L, Heslop Marshall K, Chan C, Kelly M, et al. Tailored, psychological intervention for anxiety or depression in people with chronic obstructive pulmonary disease (COPD), TANDEM (Tailored intervention for ANxiety and DEpression Management in COPD): protocol for a randomised controlled trial. Trials. 2020 Jan 6;21(1):18.

38. Curran GM, Bauer M, Mittman B, Pyne JM, Stetler C. Effectiveness-implementation hybrid designs: combining elements of clinical effectiveness and implementation research to enhance public health impact. Medical care. 2012 Mar;50(3):217-26.

39. Kelly M. SL, Sohanpal R., Pinnock H., Taylor S. The TANDEM trial: Protocol for the process evaluation of a randomised trial of a complex intervention for anxiety or depression in people living with chronic obstructive pulmonary disease. Under review.

40. Heslop-Marshall K, Carrick-Sen D, Newton J, Echevarria C, Stenton C, Jambon M, Gray J, Pearce K, Burns G, et al. . Randomised controlled trial of cognitive behavioural therapy in COPD. ERJ Open Res. 2018;4(4):00094-2018.

41. Gale NK, Heath, G., Cameron, E. et al. Using the framework method for the analysis of qualitative data in multi-disciplinary health research. BMC Med Res Methodol. 2013;13(117).

42. Borrelli B SD, Ernst D, Bellg AJ, Czajkowski S, Breger R, DeFrancesco C, Levesque C, Sharp DL, Ogedegbe G, Resnick B, Orwig D. A new tool to assess treatment fidelity and evaluation of treatment fidelity across 10 years of health behavior research. J Consult Clin Psychol. 2005;73(5):852-60.

43. Eldridge SM, Campbell MJ, Thabane L, Hopewell S, Coleman CL, et al. Defining Feasibility and Pilot Studies in Preparation for Randomised Controlled Trials: Development of a Conceptual Framework. PLoS ONE. 2016;11(3):e0150205.

44. Heslop K, Baker C, Burns G, Carrick-Sen D, De Soyza A. Effectiveness of cognitive behavioural therapy (CBT) interventions for anxiety in patients with chronic obstructive pulmonary disease (COPD) 
undertaken by respiratory nurses: the COPD CBT CARE study: BMC pulmonary medicine. 2013;13.

45. Apps LD MK, Harrison SL, Sewell L, Williams JE, Young HM, et al. The development and pilot testing of the Self-management Programme of Activity, Coping and Education for Chronic Obstructive Pulmonary Disease (SPACE for COPD). International Journal of COPD. 2013;8:317-27.

46. Rothman S. Pilling. The competencies required to deliver effective cognitive and behavioural therapy for people with depression and with anxiety disorders. UCL, 2007.

47. Beck AT. The diagnosis and management of depression. Philadelphia, PA: University of Pennsylvania Press; 1967.

48. Bandura A . Social Learning Theory. Englewood Cliffs, NJ: Prentice Hall; 1977.

49. Bandura A. Self-efficacy: the exercise of control. New York:NY: Freeman; 1997.

50. Leventhal L, Leventhal E.. Representations, procedures and affect in illness self regulation. . In: Baum A RT, Singer J, editor. Handbook of Health Psychology. New York: Erlbaum; 1997.

51. Fleming N, Helping Students Understand How They Learn. The Teaching Professor,.1992;7(4).

52. Yardley L., Arden-Close E. \& Muller I. The person-based approach to enhancing the acceptability and feasibility of interventions. Pilot and feasibility studies. 2015;1:37.

53. Cumbria N, Tyne and Wear NHS Foundation Trust. Self Help Leaflets. Available from: https:// web.ntw.nhs.uk/selfhelp - accessed 15.05.16.

54. Clark DM, Smithies R., Richards DA., Suckling R., Wright B. Improving access to psychological therapy: Initial evaluation of two UK demonstration sites. Behaviour Research and Therapy. 2009;47(11):910-20.

55. Czajkowski S, Powell LH., Adler N., Naar-King S., Reynolds KD., Hunter C., Laraia B., Olster DH., Perna FM., Peterson JC., Epel E., Boyington JE., Charlson ME. From Ideas to Efficacy: The ORBIT Model for Developing Behavioral Treatments for Chronic Diseases. Health Psychol. 2015;34(10):971-82.

56. Luszczynska A. It's time for effectiveness- implementation hybrid research on behaviour change, Health Psychology Review. 2020

57. Physicians RCo. National Asthma and COPD Audit Programme (NACAP): pulmonary rehabilitation workstream 2018 [cited 2020 8th May]. Available from:

https://http://www.rcplondon.ac.uk/projects/national-asthma-and-copd-audit-programme-nacappulmonary-rehabilitation-workstream.

58. (NHS) NHS. The NHS long term plan 2019. Available from: https://http://www.longtermplan.nhs.uk.

\section{Tables}

Table One:- Guiding Principles for TANDEM 


\section{Intervention Guiding Principles}

Depression and anxiety are key topics but could be introduced via breathlessness

The Intervention should be tailored/flexible to individuals

\section{Illustrating Quotes}

I think they most often talk about symptoms like breathlessness, rather than saying that they're anxious or depressed. (HCP006 Physio)

"terminology is important" such as "dealing with" "living with" (patient)

it's just that patients are all different, and therefore present very differently and the intervention has to be tailored individually to what they're presenting with. (HCP003, psychologist)

So I think having the capacity to start off at home is certainly a good idea. I think just something about accessible locations. (HCP002, Psychologist) or clinic but there may be limitations to the latter, accessibility is key

Clear expectations and boundaries should be set at the start of the intervention

\section{Implementation Guiding Principles Illustrating quotes}

Delivery by Respiratory

Professionals rather than psychologists is preferable

Some selection and training of facilitators will be needed
So there needs to be quite clear boundaries about what the intervention offers and doesn't offer.(HCP006, physio)

"it feels important that other members of the healthcare team are being trained up in these approaches. That can only be a good thing.... (HCP003,psychologist)

A lot of people would be attracted to this, but it's not for everyone to deliver.(HCP005, physio)

'What training would this nurse have?' (Patient)

Supervision of facilitators delivering the intervention is I think that's important.[supervision] (HCP005, physiotherapist) essential and should be ongoing

The intervention must be deliverable and supported by management

The intervention should be able to account for patient breaks due to illness
'There's no point evaluating it if it's not something that's going to be deliverable. (HCP FG001 Doctor)

Table 2: Summary of TANDEM intervention (patient facing) content 


\begin{tabular}{|c|c|c|}
\hline & Topics Covered & Content \\
\hline \multirow[t]{4}{*}{$\begin{array}{l}\text { Session } \\
1\end{array}$} & $\begin{array}{l}\text { Introduction, } \\
\text { setting } \\
\text { expectations }\end{array}$ & $\begin{array}{l}\text { Eliciting the patients understanding of COPD, identifying and } \\
\text { working with illness and treatment beliefs and acceptance. } \\
\text { Teaching basic breathing control. }\end{array}$ \\
\hline & $\begin{array}{l}\text { Topic 1- What is } \\
\text { COPD? }\end{array}$ & \\
\hline & $\begin{array}{l}\text { Topic } 2 \text { - Taking } \\
\text { control of COPD }\end{array}$ & \\
\hline & $\begin{array}{l}\text { Topic } 3 \text { - The } \\
\text { patient experience } \\
\text { of breathlessness }\end{array}$ & \\
\hline \multirow[t]{2}{*}{$\begin{array}{l}\text { Session } \\
2\end{array}$} & $\begin{array}{l}\text { Feedback from } \\
\text { home practice }\end{array}$ & $\begin{array}{l}\text { Conducting a formulation and presentation of a cognitive } \\
\text { behavioural approach }\end{array}$ \\
\hline & $\begin{array}{l}\text { Topic } 4- \\
\text { Introducing mood } \\
\text { and COPD }\end{array}$ & \\
\hline \multirow[t]{4}{*}{$\begin{array}{l}\text { Session } \\
3-7\end{array}$} & $\begin{array}{l}\text { Feedback from } \\
\text { home practice }\end{array}$ & $\begin{array}{l}\text { Up to four sessions to conduct cognitive behavioural work on } \\
\text { anxiety and/or depression dependent on individual need. One } \\
\text { f unther cescion }\end{array}$ \\
\hline & $\begin{array}{l}\text { Topic } 5 \text { - } \\
\text { Managing Anxiety } \\
\text { and COPD }\end{array}$ & \\
\hline & $\begin{array}{l}\text { Topic } 6- \\
\text { Managing } \\
\text { Depression and } \\
\text { COPD }\end{array}$ & \\
\hline & $\begin{array}{l}\text { Topic } 7 \text { - Applying } \\
\text { the CBA to other } \\
\text { problems } \\
\text { (optional) }\end{array}$ & \\
\hline \multirow[t]{2}{*}{$\begin{array}{l}\text { Session } \\
5-7\end{array}$} & $\begin{array}{l}\text { Feedback from } \\
\text { home practice }\end{array}$ & $\begin{array}{l}\text { Self-management approaches to COPD. Learning to problem solve } \\
\text { and set goals. }\end{array}$ \\
\hline & $\begin{array}{l}\text { Topic } 8 \text { - Living } \\
\text { with COPD day to } \\
\text { day }\end{array}$ & \\
\hline \multirow[t]{2}{*}{$\begin{array}{l}\text { Session } \\
6-8\end{array}$} & $\begin{array}{l}\text { Feedback from } \\
\text { home practice }\end{array}$ & Expectations of PR, addressing worries and concerns \\
\hline & $\begin{array}{l}\text { Topic } 9 \text { - } \\
\text { Preparing for } \\
\text { Pulmonary } \\
\text { Rehabilitation }\end{array}$ & \\
\hline
\end{tabular}


Table Three: Overview of TANDEM facilitator training.

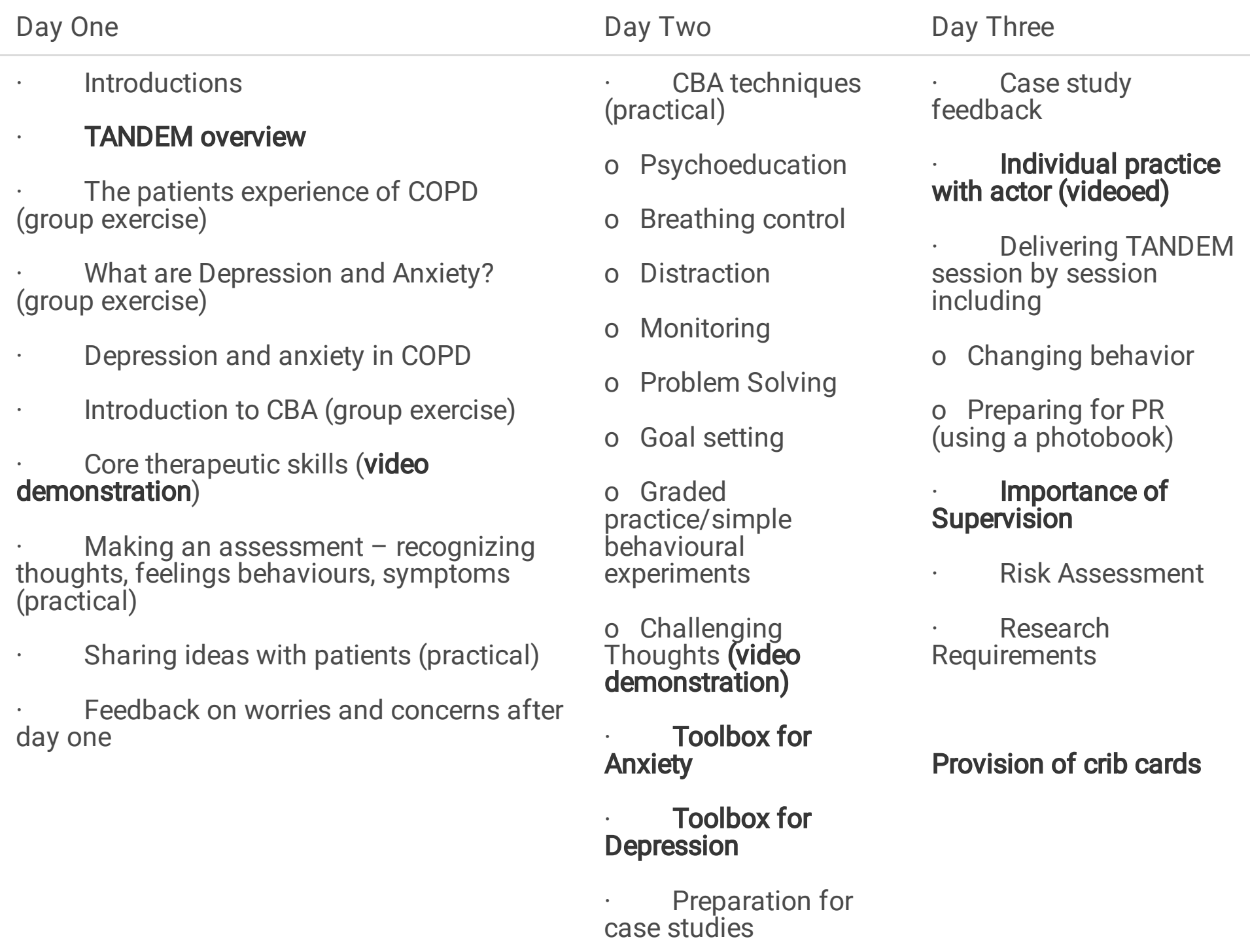

Bold typeface represents additions to the training after conducting the real world pre-pilot.

\section{Figures}




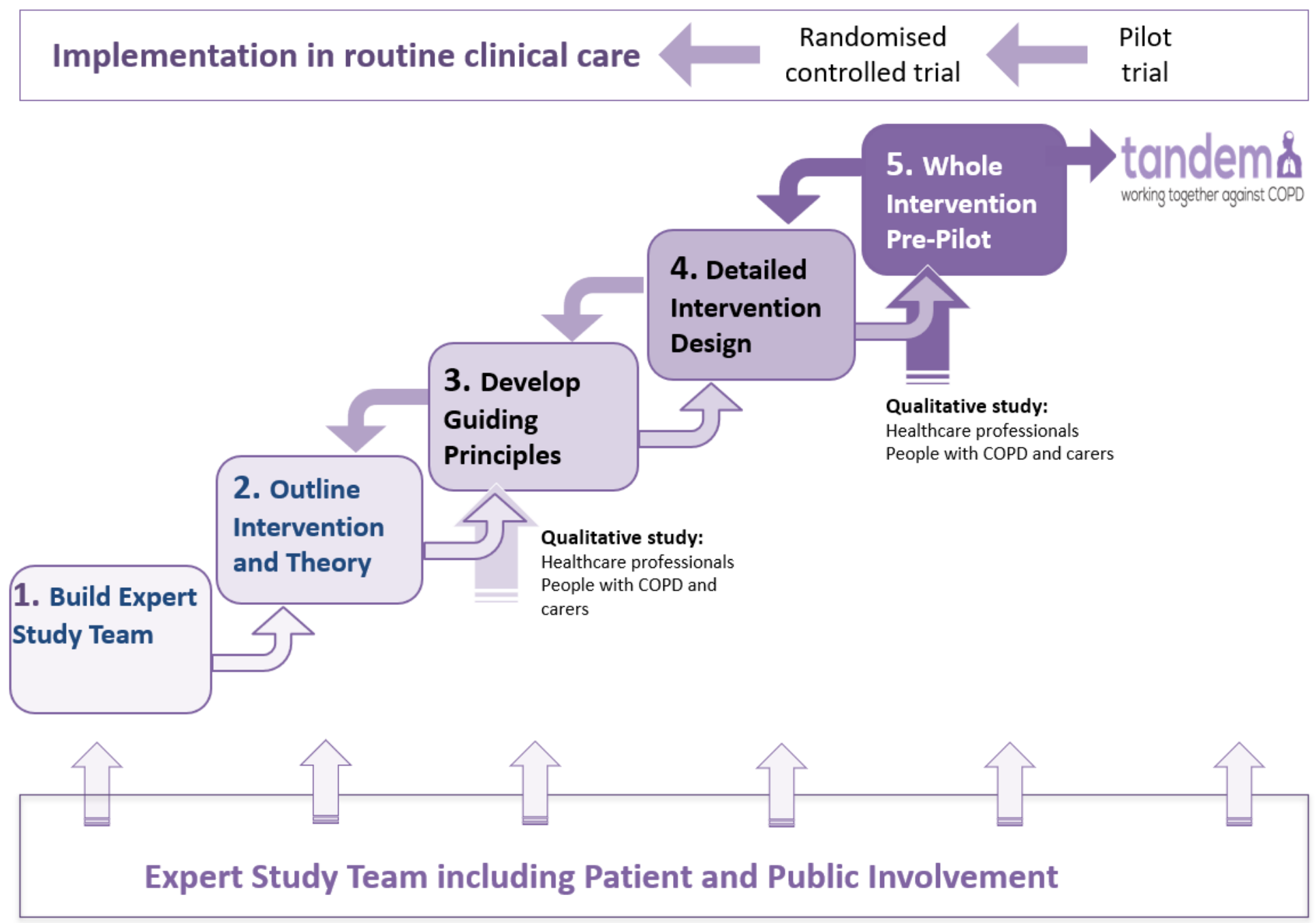

Figure 1

Schema for TANDEM Intervention Development Process 


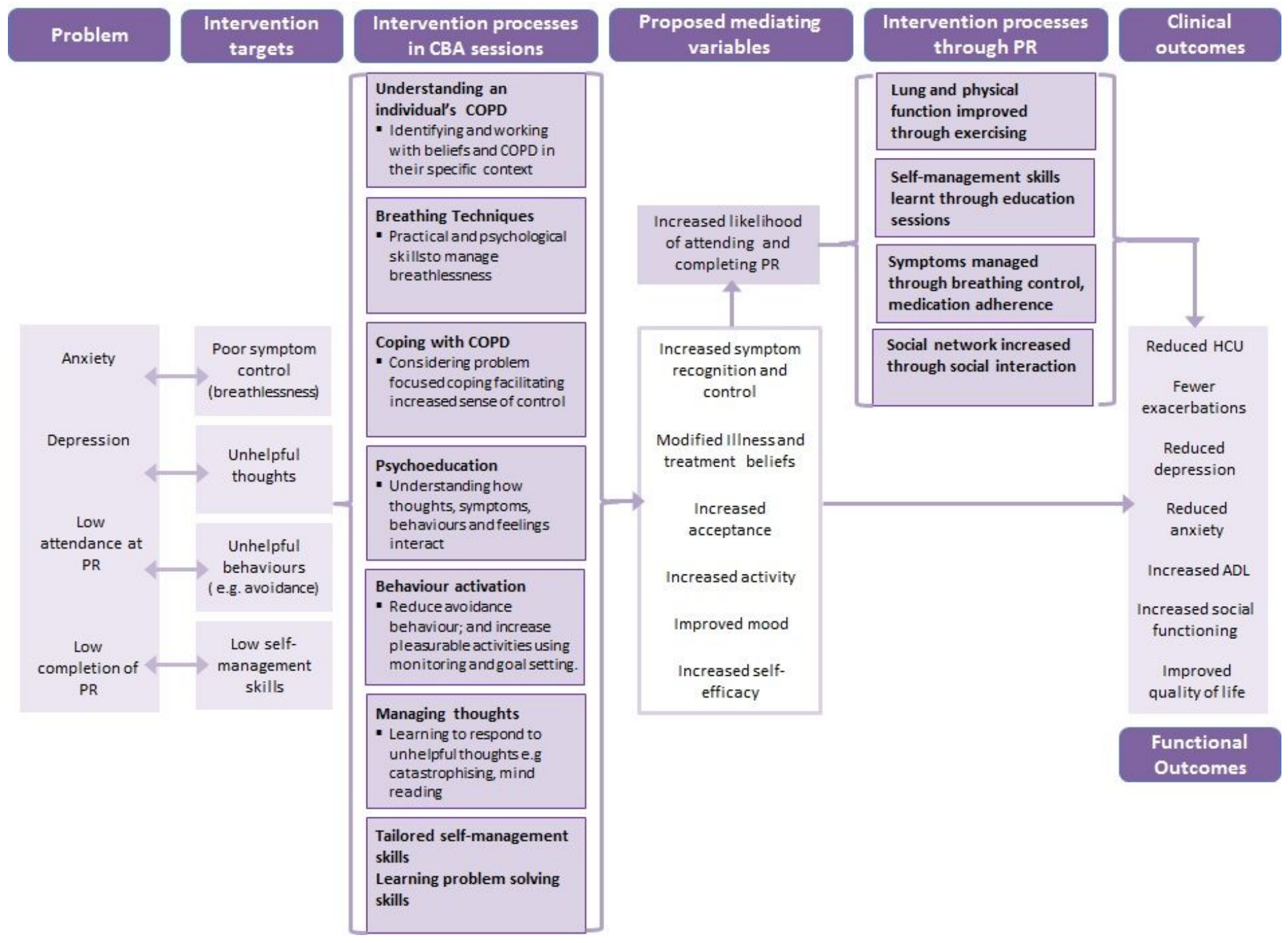

Figure 2

Logic Model for TANDEM Intervention

\section{Supplementary Files}

This is a list of supplementary files associated with this preprint. Click to download.

- SteedTANDEMAdditionalFile1Tidier.docx

- STEEDGuidedpg2.pdf

- STEEDGUIDEDPG1.pdf

- SupplementaryMaterial10utlineintervention.docx

- SupplementaryMaterial2feedback.docx 